\title{
Cortical potentials evoked by deep brain stimulation in the subthalamic area
}

\author{
Annaelle Devergnas ${ }^{1 *}$ and Thomas Wichmann ${ }^{1,2}$ \\ Wichmann Lab, Division of Neuropharmacology and Neurologic Diseases, Yerkes National Primate Research Center, Emory University, Atlanta, GA, USA \\ Department Neurology, School of Medicine; Emory University, Atlanta, GA, USA
}

Edited by:

James M. Tepper, Rutgers, The State University of New Jersey, USA

\section{Reviewed by:}

Gordon Arbuthnott, Okinawa Institute

of Science and Technology, Japan

Philip Starr, University of California

San Francisco, USA

\section{*Correspondence.}

Annaelle Devergnas, Yerkes National

Primate Research Center, Emory

University, 954 Gatewood Road NE,

Atlanta, GA 30329, USA.

e-mail: adevergnas@emory.edu

\begin{abstract}
Deep brain stimulation (DBS) of the subthalamic nucleus (STN) has been used since the mid1990s as a treatment for patients with Parkinson's disease, and more recently also in other conditions, such as dystonia or obsessive compulsive disorder. Non-invasive studies of cortical evoked potentials (EPS) that follow individual STN-DBS stimuli has provided us with insights about the conduction of the DBS pulses to the cortex. Such EPs have multiple components of different latencies, making it possible to distinguish short-latency and long-latency responses (3-8 ms and 18-25 ms latency, respectively). The available evidence indicates that these shortand long-latency EPs correspond to conduction from the STN stimulation site to the cortical recording location via anti- and orthodromic pathways, respectively. In this review we survey the literature from recording studies in human patients treated with STN-DBS for Parkinson's disease and other conditions, as well as recent animal studies (including our own) that have begun to elucidate details of the pathways, frequency dependencies, and other features of EPs. In addition, we comment on the possible clinical utility of this knowledge.
\end{abstract}

Keywords: cortical evoked potential, deep brain stimulation, subthalamic nucleus

\section{INTRODUCTION}

High-frequency stimulation of subcortical brain targets (deep brain stimulation, DBS), specifically of the subthalamic nucleus (STN) is an effective clinical treatment for patients with advanced Parkinson's disease. The effectiveness of DBS is currently under evaluation in other disorders as well, including intractable dystonia (Coubes et al., 2000; Vercueil et al., 2001; Yianni et al., 2003; Vidailhet et al., 2005), obsessive compulsive disorder (Cosyns et al., 2003; Gabriels et al., 2003; Nuttin et al., 2003), or epilepsy (Chabardes et al., 2002, 2008; Hodaie et al., 2002). While the mechanism of action of STN-DBS remains unclear and may differ across functional domains and disease states, authors agree that many of the effects of STN stimulation ultimately involve the modulation of the activities or excitability of the frontal cortex. This belief has prompted a large number of studies of stimulation-related changes in cortical activity patterns. Thus, imaging studies of the effects of STN-DBS have provided unique insight into slow, large-scale changes in cortical activity (Carbon and Eidelberg, 2002), while studies of electrophysiological changes induced by STN-DBS have provided data with higher spatial and temporal resolution. In this review, we review the literature on cortical evoked potentials (EPs) induced by STN-DBS, and supplement this material with some of our own primate recording studies.

\section{RELEVANT CIRCUIT ANATOMY}

Knowledge of the anatomy of the circuit elements by which the basal ganglia are connected to regions of the thalamus and cortex is important for an understanding of the mechanisms that are involved in EP generation after electrical stimulation of the STN. As shown in Figure 1, the STN is located in a very crowded region of the brain. Only some of the major pathways that may contribute to the generation of EPs are shown in Figure 1.
The STN is part of the "indirect" pathway of the basal ganglia. This pathway links the principal input structure of the basal ganglia, the striatum, to the output structures, the internal segment of the globus pallidus (GPi), and the substantia nigra pars reticulata $(\mathrm{SNr})$, via the external segment of the globus pallidus $(\mathrm{GPe})$ and the STN. As part of this pathway, the STN receives GPe inputs, and sends glutamatergic (excitatory) projections to both segments of the globus pallidus and to the SNr. Projections from GPi and SNr are directed to the thalamus (ventral anterior, ventral lateral, and intralaminar nuclei). Thalamic efferents are then directed back to the frontal cortex.

In addition to the indirect pathway connections from the GPe, the STN receives direct cortical inputs. The existence of this projection has been documented several decades ago in rats and monkeys (Monakow et al., 1978; Bevan et al., 1995; Nambu et al., 2000), but the exact anatomical origin(s) of this projection are still debated. Anatomical and electrophysiological studies in rats have indicated that cortical afferents to the STN originate principally from neurons in layer $\mathrm{V}$ of the cerebral cortex, with at least some arising as collaterals of corticospinal projections (Donoghue and Kitai, 1981; Kitai and Deniau, 1981; Giuffrida et al., 1985). Cortical stimulation studies in rats have suggested that the cortico-subthalamic projection arises from both the ipsilateral and contralateral cortex (Rouzaire-Dubois and Scarnati, 1985), and that responses of STN neurons to stimulation of the contralateral cortex can be eliminated by sectioning the corpus callosum (Fujimoto and Kita, 1993). Primate studies, in contrast, have indicated that the corticosubthalamic projection is ipsilateral, and arises from more circumscribed cortical areas (Monakow et al., 1978; Carpenter et al., 1981; Jurgens, 1984; Matsumura et al., 1992; Nambu et al., 1996). In monkeys, projections from the primary motor cortex were shown to terminate in the dorsolateral motor portion of the STN. The 


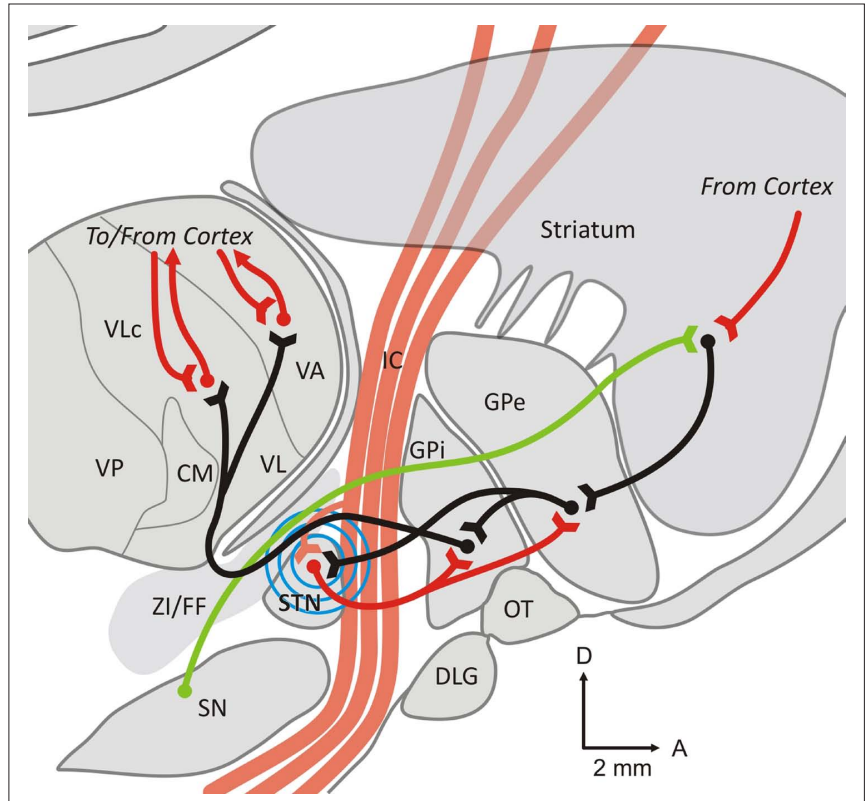

FIGURE 1 | Parasagittal slice through the monkey brain at the L7 level.

The figure shows some of the major anatomical pathways that are affected by STN stimulation, and may contribute to the generation of cortical evoked potentials. Excitatory (glutamatergic) pathways are shown as red lines, inhibitory (GABAergic) connections are shown as black lines, and modulatory dopaminergic fibers as green lines. The blue circles symbolize the spread of the electrical stimulation of the STN. See text for further details. Abbreviations: CM, centromedian nucleus of the thalamus; DLG, lateral geniculate body; FF, Fields of Forel; IC, internal capsule; GPe, external pallidal segment; GPi, internal pallidal segment; OT, optic tract; Put, putamen; SN, substantia nigra; STN, subthalamic nucleus; VA, ventral anterior nucleus of the thalamus; VL, ventrolateral nucleus of the thalamus; ZI, zona incerta.

premotor and supplementary motor areas project also to the STN but target principally the ventromedial part (partly overlapping with the projection from the primary motor cortex); Region more concerned by oculomotor and associative aspects of the motor behavior (Parent and Hazrati, 1995; Nambu et al., 1996). Although this has not been examined in as much detail, non-motor areas of the frontal cortex are also likely to project to the STN (Monakow et al., 1978; Canteras et al., 1990; Maurice et al., 1998).

Because STN-DBS may not entirely respect nuclear boundaries, it is important to consider the anatomic proximity of the STN to nearby fiber tracts. Only a subset of these fiber tracts are shown in Figure 1. The STN abuts and is traversed by medial components of the internal capsule, and is in immediate proximity to the zona incerta (ZI) and the fields of Forel (dorsal to the STN), which contain a variety of fiber tracts, particularly pallido-thalamic fibers (Baron et al., 2001), as well as dopaminergic, serotonergic, and other projections from the brainstem to the striatum and other areas. In addition, cerebello-thalamic fibers travel close to the medial and posterior part of the STN (Yelnik and Percheron, 1979; Chang et al., 1983; Kita et al., 1983; Parent and Hazrati, 1995; Hamani et al., 2004).

\section{MECHANISM OF ACTION OF STN-DBS}

The currently used DBS electrodes are bundles of four macroelectrodes whose exposed tips are separated by $0.5-1.5 \mathrm{~mm}$. During DBS procedures, these electrodes are implanted into brain regions like the STN. Typically, STN-DBS electrodes are implanted into the central STN. The most ventral electrode tends to be implanted at the ventral border of the STN, or may extend into the dorsal SNr. Depending on the contact separation of the specific electrode used, the top contact is either located in the ZI or in the central thalamus. Subsequently, continuous high-frequency stimulation is delivered via an implanted pulse generator which can be externally programmed.

The exact mechanisms of action of DBS remain uncertain. Modeling studies have suggested that the electrical parameter used in clinically effective monopolar stimulation (for instance, stimulation with $130 \mathrm{~Hz}$, and a 90- $\mu$ s pulse width) affects axons within a 2.5-mm radius around the contact center (Wu et al., 2001; Mcintyre et al., 2004). Depending on the implantation location, this volume of activation includes areas beyond the borders of the STN.

Early explanations of the mechanisms by which STN-DBS produces its antiparkinsonian effects were strongly influenced by the observation that lesions of the basal ganglia output nuclei and DBS of the STN produce roughly similar clinical outcomes in patients with Parkinson's disease (Benabid, 2003), suggesting that both may act to inhibit basal ganglia output. Support for this view came from studies of neuronal activity in the STN neurons during stimulation and from recordings of the neuronal activity in the rodent $\mathrm{SNr}$ (Benazzouz et al., 1995, 2000). However, in these studies electrical stimulation artifacts prevented the analysis of recordings during the stimulation, so that only post-stimulation activities could be tested. Several of the more recent studies have used digital stimulus artifact removal techniques (Wichmann, 2000; Hashimoto et al., 2002; Montgomery et al., 2005; Erez et al., 2010), and have either confirmed that basal ganglia output is reduced by STN stimulation (Tai et al., 2003; Meissner et al., 2005; Ma et al., 2007) or shown that it is increased (Hashimoto et al., 2003; Maurice et al., 2003; Montgomery, 2006; Xu et al., 2008; Moran et al., 2010). The conclusion that STN output to the basal ganglia output nuclei may be increased is also supported by microdialysis (Windels et al., 2000, 2003, 2005) and imaging studies (fMRI and PET, see, e.g., Jech et al., 2001; Perlmutter et al., 2002; Hershey et al., 2003).

While there is no agreement on the effects of STN stimulation on the overall firing rates in the basal ganglia, the effects of STN stimulation on activity patterns in the basal ganglia are less controversial. One of the prominent findings in the available studies is that the stimulation appears to entrain basal ganglia output, thus replacing disruptive basal ganglia output with potentially less disruptive rhythmical activities which may act like a "functional lesion" (Hashimoto et al., 2003; Ma et al., 2007; Erez et al., 2009). It is important to realize that the different rate and pattern changes with STN-DBS may not be exclusive of one another. In fact, it has been proposed that STN-DBS may act by several concurrent mechanisms on different various parkinsonian signs (Temperli et al., 2003).

\section{GENERAL FEATURES OF EPs RELATED TO STN-DBS IN CLINICAL STUDIES}

Stimulation of the STN with single stimuli or with trains of stimuli produces potentials that can be detected with stimulustriggered averaging of electroencephalographic (EEG) or electrocorticographic (ECoG) signals in frontal and central cortical 
regions. Several studies have reported the presence of such cortical EPs during low-frequency STN stimulation in patients with Parkinson's disease (Limousin et al., 1998; Ashby et al., 2001; Baker et al., 2002; Mackinnon et al., 2005; Eusebio et al., 2009; Kuriakose et al., 2009).

With low-frequency stimulation of the STN, cortical EPs with short (3-8 ms) or long latencies (18-25 ms) can be distinguished. Thus, Ashby et al. (2001) reported the presence of short-latency EPs in almost all of their parkinsonian patients, while a later study found that short-latency EPs were more variable across subjects, occurring in less than half of the electrodes tested (Mackinnon et al., 2005), but that EPs at longer latency (average of $23 \mathrm{~ms}$ ) occurred more reliably (Mackinnon et al., 2005). Long-latency EPs were also described by other authors in studies which examined EPs in response to stimulation of the contact that yielded the best clinical response (Limousin et al., 1998; Eusebio et al., 2009). In these human studies, the amplitude of EPs strongly depended on the location of the DBS contact(s) used for stimulation, likely reflecting the topography of the STN and of the fiber tracts around it. In contrast, the anatomical distribution and polarity of the potentials were found to be relatively constant across cortical regions, regardless of contact choice. Short- and long-latency EPs were found as positive potentials in the ipsilateral frontal cortex and as negative potentials over the parietal cortex (Mackinnon et al., 2005). Other authors confirmed that the largest short- or long-latency EPs are found in the ipsilateral frontal and central areas (Eusebio et al., 2009; Kuriakose et al., 2009). EPs of short latency were found to be smaller than those of longer latency.

Studies of EPs evoked by STN-area stimulation at clinically used frequencies $(80-130 \mathrm{~Hz})$ are limited because the inter-stimulus interval is shorter than the latency of the long-latency EPs. Several studies have, however, examined EPs evoked by stimulation at frequencies between 5 and $30 \mathrm{~Hz}$. Using such stimulation of the electrode contact that gave the best clinical response, the amplitude of the EP was found to be frequency-dependent, with a peak at $20 \mathrm{~Hz}$ stimulation, while the latency remained constant, regardless of the stimulation frequency (Eusebio et al., 2009). A more limited comparison of the EPs evoked by STN-DBS of 3 or $30 \mathrm{~Hz}$ in parkinsonian patients did not find significant changes in EPs (Kuriakose et al., 2009).

\section{MECHANISMS OF EP GENERATION IN STN-DBS SHORT-LATENCY EPS}

Ashby et al. (2001) found that short-latency frontal cortical EPs have a short chronaxie, a short refractory period, and follow trains of stimuli up to $100 \mathrm{~Hz}$ without change in amplitude. These characteristics suggest that the short-latency EPs arise from the activation of low-threshold neuronal elements, such as myelinated axons, rather than from the activation of cell bodies. Given the short latencies, it is likely that such responses are produced by antidromic stimulation of fibers in the vicinity of the STN. The aforementioned high-frequency following is one of the features traditionally used to verify the antidromic spread of neuronal stimulation (Degos et al., 2008), and thus, consistent with the notion that short-latency responses are antidromically mediated. Antidromic transmission from a stimulation site in the STN-area to the cortex has been documented with intracellular recordings of neurons in deep cortical layers in rodents (Li et al., 2007; Gradinaru et al., 2009).
The time needed for orthodromic conduction between motor cortex and STN in non-human primates was found to be $5.8 \pm 4.5 \mathrm{~ms}$ (Nambu et al., 2000). Because of the absence of synaptic delays, antidromic conduction can be expected to be at least 1-2 ms faster in these animals, and may be slightly longer in patients. Thus, the latency found for short-latency EPs ( $3-8 \mathrm{~ms}$ in humans) is in the range of antidromically mediated potentials.

Short-latency cortical EPs induced by STN stimulation have been found in anesthetized (Li et al., 2007) and conscious rats (Dejean et al., 2009), consistent with antidromic activation of corticofugal fibers. A detailed current-source density analysis established the source of the recorded waves as layer V (Li et al., 2007). Recent optogenetic experiments in dopamine-depleted rodents also support the concept that STN stimulation has antidromically mediated effects on the cerebral cortex (Gradinaru et al., 2009). In these studies, activation of corticofugal fibers was found to be a prerequisite for the beneficial effects of STN stimulation.

The discussion of antidromic cortical activation focuses on the cortico-subthalamic and corticospinal pathways. It remains unclear whether these pathways are separate or whether the cortico-subthalamic projection is a collateral of the corticospinal projection. If the fiber tract(s) responsible for short-latency EPs terminate in the spinal cord, suprathreshold stimulation would be expected to result not only in the generation of EPs, but also in EMG activation which would be expected to be time-locked to the stimulus. This hypothesis has been tested in several studies. Ashby et al. (1999) found in 14 parkinsonian patients undergoing STN-DBS a short-latency facilitation of EMG in the distal and proximal upper limb muscles, with latencies shorter than those seen in transcortical magnetic stimulation induced in the same subjects. This result was interpreted as suggesting that the activation of the peripheral muscles was not dependent on transcortical transmission, but was directly transmitted from the STN stimulation site to the peripheral muscles (Ashby et al., 1999). The same conclusion was reached in a subsequent study in which the facilitation peaks in the EMG (like short-latency EPs) occurred most consistently with stimulation of ventral contacts of the electrode (Ashby et al., 2001). However, more recent studies have failed to identify EMG activation patterns that are time-locked with STN stimulation (Hanajima et al., 2004; Mackinnon et al., 2005; Kuriakose et al., 2009). The discrepancies between these studies are most easily explained by differences in the location of the stimulating electrodes, the strength of stimulation and other technical differences. The negative outcome of the more recent studies obviously suggests that STN stimulation does not always antidromically activate the corticospinal tract to the extent of activation of peripheral muscles.

In our recent electrophysiologic recording studies in monkeys (Figure 2), we compared the effects of stimulation of the STN on cortical activities with those of stimulation of the GPi. The animals were chronically implanted with EEG recording electrodes, and stimulation electrodes were acutely placed in the center of the STN or into the lateral GPi. We found that only stimulation in the STN produced short-latency cortical EPs, while stimulation at either site produced long-latency EPs (see below), suggesting that only STN stimulation, but not GPi stimulation, antidromically activated fibers related to the motor cortical recording site. 


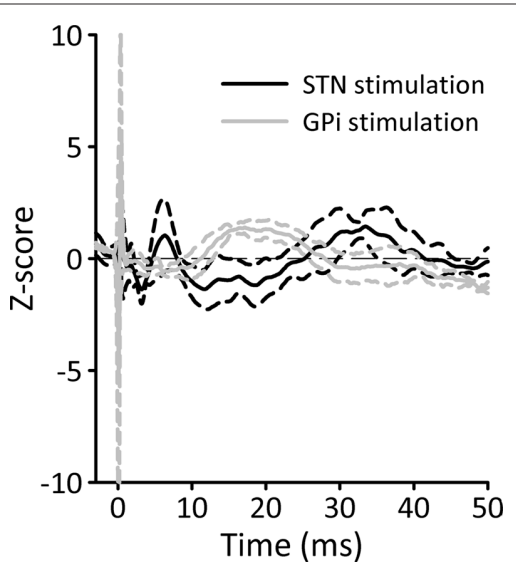

FIGURE 2 | Cortical potentials evoked by STN or GPi stimulation, recorded in an awake MPTP-treated primate. The animal was chronically implanted with two epidural EEG recording electrodes over the primary motor cortex. Single STN or GPi stimuli were delivered via a constant current source [SNEX-100 bipolar stimulation electrode (Rhoades Medical), biphasic stimulation, $10 \mathrm{~Hz}, 100 \mu \mathrm{s} /$ phase, contact separation, $0.5 \mathrm{~mm}$ ]. The figure shows EEG potential changes, averaged across 100 stimulation trials. The EEG was recorded with a differential montage between the two electrodes (filter settings $0.5-100 \mathrm{~Hz}$ ). Prior to averaging, peri-stimulation EEG segments were aligned to the onset of stimulation (Time $0 \mathrm{~ms}$ ). The solid lines represent the average response (black for STN and gray for GPi) and the dashed lines correspond to the SD.

It remains to be studied whether antidromic activation of fibers of passage is restricted to the motor system, or whether non-motor afferents to the STN are also antidromically activated. Recent anatomical studies have confirmed that the STN receives non-motor inputs (see above). It is possible that STN stimulation produces some of its non-motor side effects on mood, verbal fluency or response inhibition (Aron and Poldrack, 2006; Ballanger et al., 2009; Hershey et al., 2010) via antidromic activation of these non-motor pathways. Differences between GPi and STN stimulation as seen for motor cortex stimulation may also hold for the stimulation of nonmotor areas of cortex, which may, in turn, be related to the different non-motor side effect profile of the two procedures (Bronstein et al., 2011). The amplitude of short-latency EPs is typically larger with stimulation via the ventral contacts than with stimulation via the dorsal contacts (Ashby et al., 2001; Mackinnon et al., 2005). It was proposed that the most ventral contacts of the DBS electrode may be closest to myelinated passing fibers in the internal capsule which could be reached by current spread. However, this argument needs to be revisited, because modeling studies have suggested that myelinated fibers are also easily reached with stimulation of the dorsal STN (Mcintyre et al., 2004) and because the density of myelinated fibers within the lateral and rostral STN does not change significantly with the ventral or dorsal location.

Finally, it needs to be recognized that short-latency EPs after STN stimulation may also arise from antidromic transmission of the ZI. The ZI is immediately dorsal to the STN (Parent and Hazrati, 1995) and very likely to be within the area of stimulation during STN-DBS. It receives cortical input from layer V from large frontal areas (Bartho et al., 2002; Bartho et al., 2007). In rats, the heaviest projection to the ZI comes from the cingular cortex, with lesser contributions from the frontal and parietal cortex (Mitrofanis and Mikuletic, 1999). Most of the downstream projections from the ZI are bilateral, particularly with the brainstem and thalamus (Power and Mitrofanis, 2001).

\section{LONG-LATENCY EPS}

The origin of long-latency EPs is likely different from that of the short-latency EPs. The length of these latencies is not compatible with antidromic transmission from the STN to the cerebral cortex, making it likely that they are produced instead by orthodromic transmission (Limousin et al., 1998). EPs of long latency are predominately produced by dorsal STN stimulation (Mackinnon et al., 2005).

Stimulation of either GPi or STN in parkinsonian patients generates long-latency EPs at similar cortical locations in the ipsilateral fronto-central cortex (Limousin et al., 1998; Tisch et al., 2008). This supports the notion that long-latency EPs involve the activation of pallido-thalamic fibers, a feature that would be common to both stimulation sites. The pallido-thalamic pathway passes through the internal capsule, and runs through the fields of Forel, in proximity to the dorsal and rostral part STN (Hamani et al., 2004). STN-DBS could trigger the activation of these fibers either through activation of the excitatory STN efferents to the GPi, which would then secondarily activate pallido-thalamic fibers, or via direct activation of pallido-thalamic projections through current spread from the STN to the Fields of Forel (Mcintyre et al., 2004; Miocinovic et al., 2006). A contribution of the subthalamo-pallido-thalamic route is supported by studies of STN-DBS-related EPs in patients with prior pallidotomies. In some of these patients, cortical EPs were found to be smaller than expected (Baker et al., 2002). The finding of relatively slow cortical responses to GPi stimulation $(10 \mathrm{~Hz}$, 3.5-4 V, 60-90 $\mu \mathrm{s}$ ) in dystonic patients (Tisch et al., 2008) also supports the hypothesis that at least a portion of the long-latency EPs are mediated via stimulation of pallido-thalamic fibers. However, in a study by Limousin et al. (1998)_EPs evoked by STN-DBS had a shorter latency (18-20 ms) than EPs evoked by GPi stimulation (25.0-25.8 ms). In our comparisons of EPs recorded from fixed location in the motor cortex, and induced by STN or GPi stimulation (gray lines in Figure 2), the long-latency responses evoked by GPi stimulation occurred earlier than those evoked by STN stimulation (black lines in Figure 2), suggesting that STN stimulation produced its long-latency effects not via direct activation of pallido-thalamic fibers, but through activation of STN efferents to the pallidum.

Because the largest amplitude of long-latency STN-DBS-evoked EPs is found in the same cortical areas that show the largest potentials evoked by stimulation of the contralateral median nerve, the DBS-induced EP may be generated by activity in the region of the ipsilateral primary motor and sensorimotor cortex, in line with the known anatomical afferent and efferent patterns of the basal ganglia (Mackinnon et al., 2005).

Because EP characteristics seem to be relatively unaffected by dopaminergic medications, it has been suggested that the generation or propagation the EPs is (relatively) independent of dopaminergic circuit (Eusebio et al., 2009). Eusebio et al. (2009) observed that oscillatory activity following EPs may be reduced by the medication, perhaps reflecting reduced resonance of the 
cortico-basal ganglia-thalamocortical loops. A suppressing effect of STN stimulation on oscillatory cortical activities is also supported by experiments in rodents, where the amplitude of evoked cortical LFPs inversely correlated with slow oscillatory activity (Li et al., 2007). The relative independence of EPs from dopaminergic effects may only apply to parkinsonian individuals, however. STN-DBS is now carried out in an increasing number of non-parkinsonian conditions (see above). In patients with these conditions, en-passant activation of dopaminergic or serotonergic fibers could be involved in the generation of long-latency EPs. However, in a small comparative study of EPs in parkinsonian or epilepsy patients (Baker et al., 2002) there were no significant differences between the two groups. This study would be worth repeating with additional patients or other disease conditions. Moreover, it had been suggested that this long-latency EP could also be independent of basal ganglia involvement. This long-latency EP could be either attributed to a corticocortical pathway driven from the intracortical projections of the pyramids that are antidromically stimulated, or either attributed to the pyramidal branches to the thalamus, those feed back to cortex and so could drive the late response (Phillips, 1959).

Stimulation of the ZI may also have orthodromic effects on cortex, via its GABAergic projection to the ventral anterior thalamus (Bartho et al., 2002). Similar to the activation of pallido-thalamic projection, activation of ZI output induces hyperpolarization of thalamocortical neurons followed by rebound bursts (Kuriakose et al., 2009).

Finally, rodent studies have shown that projections exist between the STN and the prefrontal cortex, providing yet another potential orthodromic route of transmission (Jackson and Crossman, 1981; Kita and Kitai, 1987; Degos et al., 2008). However, it is not clear whether this projection exists in primates.

\section{ACTIVITY OF CIRCUITS DOWNSTREAM FROM THE STIMULATION SITE}

Activation of the pallido-thalamic pathway by STN-DBS may alter the neuronal activity in the nuclei of the ventral and intralaminar thalamus that receive basal ganglia input (Anderson et al., 2003; $\mathrm{Xu}$ et al., 2008). As an example, Mackinnon et al. (2005) have proposed that low-frequency stimulation of the STN may lead to an inhibitory volley along pallido-thalamic pathways that may hyperpolarize ventral thalamic neurons and de-inactivate somatic calcium conductances which would then result in rebound bursting activity of thalamocortical neurons. The excitatory rebound activity may lead to the production of a cortical evoked potential in related cortical areas (Sherman, 2001; Mackinnon et al., 2005). Due to the slow kinetics of low-threshold calcium bursting activities, clinically effective high-frequency stimulation of the STN would simply lead to suppression of thalamocortical activities by inducing a hyperpolarization state during the stimulation, resulting in a net suppression of the activity of cortical neurons. In support of this idea, thalamic activity was found to be reduced during highfrequency stimulation of the GPi (Anderson et al., 2003), and a decrease in cerebral blood flow was found in the primary motor cortex of parkinsonian patients, during STN stimulation (CeballosBaumann et al., 1999; Payoux et al., 2004). However, modeling studies have suggested that high-frequency STN stimulation may induce more complex changes in thalamic firing activities (Guo et al., 2008). Of course, the low-pass filter characteristics of the recording conditions, dictated by the size of the electrodes and the dielectric properties of the scalp and other tissue elements, further contribute to the temporal dynamics of the recordings (similar to its contribution to the frequency of EEG responses).

The thalamus, finally, projects to the frontal cortex. Layer IV is the main target of thalamocortical afferents, and would, thus, be the primary cortical layer affected by orthodromically mediated effects of STN-DBS. Large pyramidal cells in layer V are the principal sources of subcortical efferents, and would be considered the primary cortical elements activated via antidromic stimulation effects, as has been shown in cats (Phillips, 1959; Giuffrida et al., 1985) and in rats (Li et al., 2007).

In addition to the latency, the durations of EPs are an interesting feature of these potentials. In our primate recording study, shortlatency potential after STN-DBS stimulation lasted 7.2 $\pm 0.4 \mathrm{~ms}$ while long-latency potentials had a total duration of $30.5 \pm 3.5 \mathrm{~ms}$ (Figure 2). The temporal spread after short stimuli goes beyond the variability of transmission delays at single synapses, suggesting that the stimulation may involve more than one pathway of origin, or that it may affect thalamic or cortical networks of connections which may eventually contribute to the temporally spread-out cortical response.

\section{POTENTIAL CLINICAL RELEVANCE}

It remains an open question whether EPs evoked by STN-DBS are related to clinically relevant phenomena. One possibility is that cortical EPs may reflect changes in cortical excitability. In support of this idea, Ashby et al. (1999) found that short-latency EPs were facilitated by paired stimuli at inter-stimulus intervals between 2 and $10 \mathrm{~ms}$, suggesting a cortical summation of postsynaptically generated EPs with short individual refractory periods (Ashby et al., 2001). Such cortical facilitation could also be partially reflected in increases in cerebral blood flow as have been detected in functional imaging studies (Limousin et al., 1997; Ceballos-Baumann et al., 1999; Hershey et al., 2003).

Experiments using transcranial magnetic stimulation (TMS) to probe cortical excitability have also detected changes in motor cortical excitability with multi-pulse STN-DBS in parkinsonian patients. In these experiments, the short-interval intracortical inhibition was normalized by STN stimulation, but the stimulation did not affect motor EP amplitudes or excitability thresholds (Cunic et al., 2002; Pierantozzi et al., 2002). These summation effect may not be the only mechanism by which STN-DBS may alter cortical activation, because short-latency facilitation of motor cortex excitability can also be seen with single-pulse STN stimulation in patients with Parkinson's disease, specifically with TMS protocols that activate corticospinal neurons through cortical interneurons (Hanajima et al., 2004; Kuriakose et al., 2009). One of the key differences between these studies and the earlier paired-pulse experiments is that the TMS stimuli and the STN-DBS were not time-locked in the paired-pulse studies, so that the temporal evolution of facilitation after each STN stimulus could not be examined. In recent studies Kuriakose et al. (2009) noted changes in motor cortex excitability only at specific time intervals after STN stimulation, corresponding to the duration of short and medium EP peaks. These findings suggest that the cortical facilitation found by TMS was linked to the occurrence of the EP induced by STN stimulation. 
Although the antiparkinsonian effects of TMS are generally considered to be moderate compared to those of DBS (Fregni et al., 2005). Similar to the DBS effects (Benabid, 2003; Benabid et al., 2005), the frequency-dependence of the two treatments appears to be similar: low-frequency TMS does not produce beneficial effects (Arias et al., 2010), while high frequency TMS may produce motor benefits (Elahi and Chen, 2009).

Several authors have studied whether the presence or amplitude of EPs could be used as an intraoperative guide to optimize placement of DBS electrode. This would be beneficial, particularly in cases in whom the therapeutic effects of STN-DBS are not immediately apparent (such as in patients with dystonia, epilepsy, depression, or OCD). This approach appears to work in cases of GPi-DBS in dystonic patients (Tisch et al., 2008), but in the case of STN-DBS, the short- or long-latency components of EPs do not appear to strongly correlate with the clinical outcome of the surgery (Ashby et al., 1999; Mackinnon et al., 2005). A principal problem with these studies is that EP testing was done with stimulation at frequencies below $30 \mathrm{~Hz}$ (see above) to examine long-latency EPs. At least in Parkinson's disease patients, these stimulation conditions are not optimal from a clinical perspective (Benabid, 2003) and may, in fact, limit the usefulness of intraoperative EP testing as a targeting tool to identify clinical useful stimulation sites. Interestingly, a recent study has indicated that the clinically effective contacts may correspond to those that produce long-latency EPs when tested at a stimulation frequency of $10 \mathrm{~Hz}$ (Kuriakose et al., 2009). The utility of short-latency EPs has not been fully evaluated in this context, but studies in awake rodents treated with dopamine receptor blockers documented a correlation between the amplitude of short-latency cortical activation and the degree of improvement in akinesia (Dejean et al., 2009).

\section{REFERENCES}

Anderson, M.E., Postupna, N., and Ruffo, M. (2003). Effects of high-frequency stimulation in the internal globus pallidus on the activity of thalamic neurons in the awake monkey. $J$. Neurophysiol. 89, 1150-1160.

Arias, P., Vivas, J., Grieve, K. L., and Cudeiro, J. (2010). Controlled trial on the effect of 10 days low-frequency repetitive transcranial magnetic stimulation (rTMS) on motor signs in Parkinson's disease. Mov. Disord. 25, 1830-1838.

Aron, A. R., and Poldrack, R. A. (2006). Cortical and subcortical contributions to Stop signal response inhibition: role of the subthalamic nucleus. J. Neurosci. 26, 2424-2433.

Ashby, P., Kim, Y. J., Kumar, R., Lang, A. E., and Lozano, A. M. (1999). Neurophysiological effects of stimulation through electrodes in the human subthalamic nucleus. Brain 122(Pt 10), 1919-1931.

Ashby, P., Paradiso, G., Saint-Cyr, J. A., Chen, R., Lang, A. E., and Lozano, A. M. (2001). Potentials recorded at the scalp by stimulation near the

\section{CONCLUSION}

Measuring cortical EPs provides us with a unique opportunity to non-invasively monitor with high temporal resolution the effects of DBS on the cerebral cortex. However, the mechanisms that are involved in generating these potential changes remain relatively poorly understood. The available information suggests that STNDBS affects multiple anatomical routes which alter cortical activities with different latencies from the stimulation.

Cortical potentials evoked by STN-DBS may not simply be epiphenomena of the stimulation, but may reflect the temporal progression of changes in cortical excitability which may be related to the clinical effects of DBS therapy in Parkinson's disease patients. However, the details of the relationship between the EPs and the clinical effects are not clear. For instance, if these potentials reflect specific activity changes at the cortical level, it is conceivable that the apparent need for high-frequency stimulation for a beneficial effects in patients with Parkinson's disease may arise from the need to optimize the interplay between short- and long-latency effects of the stimulation (or avoid long-latency potentials altogether through rapidly resetting the cortical response through short-latency responses).

We have yet to learn how to utilize the EP results to optimize the clinical outcome of patients who are being treated with STN-DBS. More study of the utility of intraoperative EP recordings to verify electrode placement, or of postoperative EP recordings as an aide to more rapid programming of the STN stimulator is needed to fully examine the potential of this technique. Animal experiments combining pharmacological or lesioning techniques to study the contribution of different anatomical routes to the stimulation response would be useful to further our understanding of the generation of EPs, and of the processes they reflect, both at the cortical and subcortical levels.

Benabid, A. L. (2003). Deep brain stimulation for Parkinson's disease. Curr. Opin. Neurobiol. 13, 696-706.

Benabid, A. L., Chabardes, S., and Seigneuret, E. (2005). Deep-brain stimulation in Parkinson's disease: long-term efficacy and safety - What happened this year? Curr. Opin. Neurol. 18, 623-630.

Benazzouz, A., Gao, D. M., Ni, Z. G., Piallat, B., Bouali-Benazzouz, R., and Benabid, A. L. (2000). Effect of high-frequency stimulation of the subthalamic nucleus on the neuronal activities of the substantia nigra pars reticulata and ventrolateral nucleus of the thalamus in the rat. Neuroscience 99, 289-295.

Benazzouz, A., Piallat, B., Pollak, P., and Benabid, A. L. (1995). Responses of substantia nigra pars reticulata and globus pallidus complex to high frequency stimulation of the subthalamic nucleus in rats: electrophysiological data. Neurosci. Lett. 189, 77-80.

Bevan, M. D., Francis, C. M., and Bolam, J. P. (1995). The glutamate-enriched cortical and thalamic input to neurons in the subthalamic nucleus of the rat: convergence with GABA-positive terminals. J. Comp. Neurol. 361, 491-511.

Bronstein, J. M., Tagliati, M., Alterman, R. L., Lozano, A. M., Volkmann, J., Stefani, A., Horak, F. B., Okun, M. S., Foote, K. D., Krack, P., Pahwa, R., Henderson, J. M., Hariz, M. I., Bakay, R. A., Rezai, A., Marks, W. J., Jr., Moro, E., Vitek, J. L., Weaver, F. M., Gross, R. E., and Delong, M. R. (2011). Deep brain stimulation for Parkinson disease: an expert consensus and review of key issues. Arch. Neurol. 68, 165.

Canteras, N. S., Shammah-Lagnado, S. J., Silva, B. A., and Ricardo, J. A. (1990). Afferent connections of the subthalamic nucleus: a combined retrograde and anterograde horseradish peroxidase study in the rat. Brain Res. 513, 43-59.

Carbon, M., and Eidelberg, D. (2002). Modulation of regional brain function by deep brain stimulation: studies with positron emission tomography. Curr. Opin. Neurol. 15, 451-455.

Carpenter, M. B., Carleton, S. C., Keller, J. T., and Conte, P. (1981). Connections of the subthalamic nucleus in the monkey. Brain Res. 224, 1-29. 
Ceballos-Baumann, A. O., Boecker, H., Bartenstein, P., Von Falkenhayn, I., Riescher, H., Conrad, B., Moringlane, J. R., and Alesch, F. (1999). A positron emission tomographic study of subthalamic nucleus stimulation in Parkinson disease: enhanced movement-related activity of motor-association cortex and decreased motor cortex resting activity. Arch. Neurol. 56, 997-1003.

Chabardes, S., Kahane, P., Minotti, L., Koudsie, A., Hirsch, E., and Benabid, A. L. (2002). Deep brain stimulation in epilepsy with particular reference to the subthalamic nucleus. Epileptic Disord. 4(Suppl. 3), S83-S93.

Chabardes, S., Minotti, L., Chassagnon, S., Piallat, B., Torres, N., Seigneuret, E., Vercueil, L., Carron, R., Hirsch, E., Kahane, P., and Benabid, A. L. (2008). Basal ganglia deep-brain stimulation for treatment of drug-resistant epilepsy: review and current data. Neurochirurgie 54, 436-440.

Chang, H. T., Kita, H., and Kitai, S. T. (1983). The fine structure of the rat subthalamic nucleus: an electron microscopic study. J. Comp. Neurol. 221, 113-123.

Cosyns, P., Gabriels, L., and Nuttin, B. (2003). Deep brain stimulation in treatment refractory obsessive compulsive disorder. Verh. K. Acad. Geneeskd. Belg. 65, 385-399; discussion 399-400.

Coubes, P., Roubertie, A., Vayssiere, N., Hemm, S., and Echenne, B. (2000). Treatment of DYT1-generalised dystonia by stimulation of the internal globus pallidus. Lancet 355, 2220-2221.

Cunic, D., Roshan, L., Khan, F. I., Lozano, A. M., Lang, A. E., and Chen, R. (2002). Effects of subthalamic nucleus stimulation on motor cortex excitability in Parkinson's disease. Neurology 58, 1665-1672.

Degos, B., Deniau, J. M., Le Cam, J., Mailly, P., and Maurice, N. (2008). Evidence for a direct subthalamo-cortical loop circuit in the rat. Eur. J. Neurosci. 27, 2599-2610.

Dejean, C., Hyland, B., and Arbuthnott, G. (2009). Cortical effects of subthalamic stimulation correlate with behavioral recovery from dopamine antagonist induced akinesia. Cereb. Cortex 19, 1055-1063.

Donoghue, J. P., and Kitai, S. T. (1981). A collateral pathway to the neostriatum from corticofugal neurons of the rat sensory-motor cortex: an intracellular HRP study. J. Comp. Neurol. 201, 1-13.

Elahi, B., and Chen, R. (2009). Effect of transcranial magnetic stimulation on Parkinson motor function systematic review of controlled clinical trials. Mov. Disord. 24, 357-363.
Erez, Y., Czitron, H., Mccairn, K., Belelovsky, K., and Bar-Gad, I. (2009). Short-term depression of synaptic transmission during stimulation in the globus pallidus of 1-methyl-4-phenyl1,2,3,6-tetrahydropyridine-treated primates. J. Neurosci. 29, 7797-7802.

Erez, Y., Tischler, H., Moran, A., and BarGad, I. (2010). Generalized framework for stimulus artifact removal. $J$. Neurosci. Methods 191, 45-59.

Eusebio, A., Pogosyan, A., Wang, S., Averbeck, B., Gaynor, L. D., Cantiniaux, S., Witjas, T., Limousin, P., Azulay, J. P., and Brown, P. (2009). Resonance in subthalamo-cortical circuits in Parkinson's disease. Brain 132, 2139-2150.

Fregni, F., Simon, D. K., Wu, A., and Pascual-Leone, A. (2005). Noninvasive brain stimulation for Parkinson's disease: a systematic review and meta-analysis of the literature.J. Neurol. Neurosurg. Psychiatr. 76, 1614-1623.

Fujimoto, K., and Kita, H. (1993). Response characteristics of subthalamic neurons to the stimulation of the sensorimotor cortex in the rat. Brain Res. 609, 185-192.

Gabriels, L., Cosyns, P., Nuttin, B., Demeulemeester, H., and Gybels, J. (2003). Deep brain stimulation for treatment-refractory obsessivecompulsive disorder: psychopathological and neuropsychological outcome in three cases. Acta Psychiatr. Scand. 107, 275-282.

Giuffrida, R., Li Volsi, G., Maugeri, G., and Perciavalle, V. (1985). Influences of pyramidal tract on the subthalamic nucleus in the cat. Neurosci. Lett. 54 231-235.

Gradinaru, V., Mogri, M., Thompson, K. R., Henderson, J. M., and Deisseroth, K. (2009). Optical deconstruction of parkinsonian neural circuitry. Science 324, 354-359.

Guo, Y., Rubin, J. E., Mcintyre, C. C., Vitek, J. L., and Terman, D. (2008). Thalamocortical relay fidelity varies across subthalamic nucleus deep brain stimulation protocols in a data-driven computational model. J. Neurophysiol. 99, 1477-1492.

Hamani, C., Saint-Cyr, J. A., Fraser, J., Kaplitt, M., and Lozano, A. M. (2004). The subthalamic nucleus in the context of movement disorders. Brain 127, 4-20.

Hanajima, R., Ashby, P., Lozano, A. M., Lang, A. E., and Chen, R. (2004). Single pulse stimulation of the human subthalamic nucleus facilitates the motor cortex at short intervals. J. Neurophysiol. 92, 1937-1943.

Hashimoto, T., Elder, C. M., Okun, M. S., Patrick, S. K., and Vitek, J. L. (2003).
Stimulation of the subthalamic nucleus changes the firing pattern of pallidal neurons. J. Neurosci. 23 , 1916-1923.

Hashimoto, T., Elder, C. M., and Vitek, J. L. (2002). A template subtraction method for stimulus artifact removal in high-frequency deep brain stimulation. J. Neurosci. Methods 113 181-186.

Hershey, T., Campbell, M. C., Videen, T. O., Lugar, H. M., Weaver, P. M. Hartlein, J., Karimi, M., Tabbal, S. D. and Perlmutter, J. S. (2010). Mapping Go-No-Go performance within the subthalamic nucleus region. Brain 133, 3625-3634.

Hershey, T., Revilla, F. J., Wernle, A. R., Mcgee-Minnich, L., Antenor, J. V., Videen, T. O., Dowling, J. L., Mink, J. W., and Perlmutter, J. S. (2003) Cortical and subcortical blood flow effects of subthalamic nucleus stimulation in PD. Neurology 61, 816-821.

Hodaie, M., Wennberg, R. A., Dostrovsky, J. O., and Lozano, A. M. (2002). Chronic anterior thalamus stimulation for intractable epilepsy. Epilepsia $43,603-608$.

Jackson, A., and Crossman, A. R. (1981). Subthalamic nucleus efferent projection to the cerebral cortex. Neuroscience 6, 2367-2377.

Jech, R., Urgosik,D., Tintera,J., Nebuzelsky, A., Krasensky, J., Liscak, R., Roth, J. and Ruzicka, E. (2001). Functiona magnetic resonance imaging during deep brain stimulation: a pilot study in four patients with Parkinson's disease. Mov. Disord. 16, 1126-1132.

Jurgens, U. (1984). The efferent and afferent connections of the supplementary motor area. Brain Res. 300, 63-81.

Kita, H., Chang, H. T., and Kitai, S. T. (1983). The morphology of intracellularly labeled rat subthalamic neurons: a light microscopic analysis. J. Comp. Neurol. 215, 245-257.

Kita, H., and Kitai, S. T. (1987). Efferent projections of the subthalamic nucleus in the rat: light and electron microscopic analysis with the PHA-L method. J. Comp. Neurol. 260, 435-452.

Kitai, S. T., and Deniau, J. M. (1981). Cortical inputs to the subthalamus: intracellular analysis. Brain Res. 214, 411-415.

Kuriakose, R., Saha, U., Castillo, G., Udupa, K., Ni, Z., Gunraj, C., Mazzella, F., Hamani, C., Lang, A. E., Moro, E., Lozano, A. M., Hodaie, M., and Chen, R. (2009). The nature and time course of cortical activation following subthalamic stimulation in Parkinson's disease. Cereb. Cortex. 20, 1926-1936.

Li, S., Arbuthnott, G. W., Jutras, M. J., Goldberg, J. A., and Jaeger, D. (2007)
Resonant antidromic cortical circuit activation as a consequence of highfrequency subthalamic deep-brain stimulation. J. Neurophysiol. 98, 3525-3537.

Limousin, P., Brown, P., Marsden, L. Defebvre, L., and Rothwell, J. (1998). Evoked potentials from subthalamic nucleus, internal pallidum and thalamic stimulation in parkinsonian and postural tremor patients. J. Physiol. 509P, 176P-177P.

Limousin, P., Greene, J., Pollak, P., Rothwell, J., Benabid, A. L., and Frackowiak, R. (1997). Changes in cerebral activity pattern due to subthalamic nucleus or internal pallidum stimulation in Parkinson's disease. Ann. Neurol. 42, 283-291.

Ma, Y., Bevan, M. D., and Wichmann, T. (2007). Effects of subthalamic nucleus stimulation on neuronal activity in the internal pallidal segment in monkeys. Soc. Neurosci. Abstr.

Mackinnon, C.D.,Webb, R.M., Silberstein, P., Tisch, S., Asselman, P., Limousin, P., and Rothwell, J.C. (2005). Stimulation through electrodes implanted near the subthalamic nucleus activates projections to motor areas of cerebral cortex in patients with Parkinson's disease. Eur. J. Neurosci. 21, 1394-1402.

Matsumura, M., Kojima, J., Gardiner, T. W., and Hikosaka, O. (1992). Visual and oculomotor functions of monkey subthalamic nucleus. J. Neurophysiol. 67, 1615-1632.

Maurice, N., Deniau, J. M., Menetrey, A., Glowinski, J., and Thierry, A. M. (1998). Prefrontal cortex-basal ganglia circuits in the rat: involvement of ventral pallidum and subthalamic nucleus. Synapse 29, 363-370.

Maurice, N., Thierry, A. M., Glowinski, J., and Deniau, J. M. (2003). Spontaneous and evoked activity of substantia nigra pars reticulata neurons during high-frequency stimulation of the subthalamic nucleus. J. Neurosci. 23, 9929-9936.

Mcintyre, C. C., Mori, S., Sherman, D. L., Thakor, N. V., and Vitek, J. L. (2004). Electric field and stimulating influence generated by deep brain stimulation of the subthalamic nucleus. Clin. Neurophysiol. 115, 589-595.

Meissner, W., Leblois, A., Hansel, D., Bioulac, B., Gross, C. E., Benazzouz, A., and Boraud, T. (2005). Subthalamic high frequency stimulation resets subthalamic firing and reduces abnormal oscillations. Brain 128, 2372-2382.

Miocinovic, S., Parent, M., Butson, C. R., Hahn, P. J., Russo, G. S., Vitek, J. L., and Mcintyre,C.C. (2006).Computational analysis of subthalamic nucleus and lenticular fasciculus activation during therapeutic deep brain stimulation. J. Neurophysiol. 96, 1569-1580. 
Mitrofanis, J., and Mikuletic, L. (1999). Organisation of the cortical projection to the zona incerta of the thalamus. J. Comp. Neurol. 412, 173-185.

Monakow, K. H., Akert, K., and Kunzle, H. (1978). Projections of the precentral motor cortex and other cortical areas of the frontal lobe to the subthalamic nucleus in the monkey. Exp. Brain Res. 33, 395-403.

Montgomery, E. B. Jr. (2006). Effects of GPi stimulation on human thalamic neuronal activity. Clin. Neurophysiol. 117, 2691-2702.

Montgomery, E. B. Jr., Gale, J. T., and Huang, H. (2005). Methods for isolating extracellular action potentials and removing stimulus artifacts from microelectrode recordings of neurons requiring minimal operator intervention.J. Neurosci. Methods 144, 107-125.

Moran, A., Stein, E., Tischler, H., Belelovsky, K., and Bar-Gad, I. (2010). "Decoupling of intra-neuron oscillations and inter-neuron synchronization during high frequency stimulation of the subthalalmic nucleus," in IBAGS X.

Nambu, A., Takada, M., Inase, M., and Tokuno, H. (1996). Dual somatotopical representations in the primate subthalamic nucleus: evidence for ordered but reversed body-map transformations from the primary motor cortex and the supplementary motor area. $J$. Neurosci. 16, 2671-2683.

Nambu, A., Tokuno, H., Hamada, I., Kita, H., Imanishi, M., Akazawa, T., Ikeuchi, Y., and Hasegawa, N. (2000). Excitatory cortical inputs to pallidal neurons via the subthalamic nucleus in the monkey. J. Neurophysiol. 84, 289-300.

Nuttin, B. J., Gabriels, L., Van Kuyck, K., and Cosyns, P. (2003). Electrical stimulation of the anterior limbs of the internal capsules in patients with severe obsessive-compulsive disorder: anecdotal reports. Neurosurg. Clin. N. Am. 14, 267-274.
Parent, A., and Hazrati, L. N. (1995). Functional anatomy of the basal ganglia. II. The place of subthalamic nucleus and external pallidum in basal ganglia circuitry. Brain Res. Brain Res. Rev. 20, 128-154.

Payoux, P., Remy, P., Damier, P., Miloudi, M., Loubinoux, I., Pidoux, B., Gaura, V., Rascol, O., Samson, Y., and Agid, Y. (2004). Subthalamic nucleus stimulation reduces abnormal motor cortical overactivity in Parkinson disease. Arch. Neurol. 61, 1307-1313.

Perlmutter, J. S., Mink, J. W., Bastian, A. J., Zackowski, K., Hershey, T., Miyawaki, E., Koller, W., and Videen, T. O. (2002). Blood flow responses to deep brain stimulation of thalamus. Neurology 58, 1388-1394.

Phillips, C. G. (1959). Actions of antidromic pyramidal volleys on single Betz cells in the cat. Q. J. Exp. Physiol. Cogn. Med. Sci. 44, 1-25.

Pierantozzi, M., Palmieri, M. G., Mazzone, P., Marciani, M. G., Rossini, P. M., Stefani, A., Giacomini, P., Peppe, A., and Stanzione, P. (2002). Deep brain stimulation of both subthalamic nucleus and internal globus pallidus restores intracortical inhibition in Parkinson's disease paralleling apomorphine effects: a paired magnetic stimulation study. Clin. Neurophysiol. $113,108-113$.

Power, B. D., and Mitrofanis, J. (2001). Zona incerta: Substrate for contralateral interconnectivity in the thalamus of rats. J. Comp. Neurol. 436, 52-63.

Rouzaire-Dubois, B., and Scarnati, E. (1985). Bilateral corticosubthalamic nucleus projections: an electrophysiological study in rats with chronic cerebral lesions. Neuroscience 15, 69-79.

Sherman, S. M. (2001). Tonic and burst firing: dual modes of thalamocortical relay. Trends Neurosci. 24, 122-126.

Tai, C. H., Boraud, T., Bezard, E., Bioulac, B., Gross, C., and Benazzouz,A. (2003). Electrophysiological and metabolic evidence that high-frequency stimulation of the subthalamic nucleus bridles neuronal activity in the subthalamic nucleus and the substantia nigra reticulata. FASEB J. 17, 1820-1830.

Temperli, P., Ghika, J., Villemure, J. G., Burkhard, P. R., Bogousslavsky, J. and Vingerhoets, F. J. (2003). How do parkinsonian signs return after discontinuation of subthalamic DBS? Neurology 60, 78-81.

Tisch, S., Rothwell, J. C., Zrinzo, L., Bhatia, K. P., Hariz, M., and Limousin, P. (2008). Cortical evoked potentials from pallidal stimulation in patients with primary generalized dystonia. Mov. Disord. 23, 265-273.

Vercueil, L., Pollak, P., Fraix, V., Caputo, E., Moro, E., Benazzouz, A., Xie, J. Koudsie, A., and Benabid,A. L. (2001) Deep brain stimulation in the treatment of severe dystonia. J. Neurol. 248 , 695-700.

Vidailhet, M., Vercueil, L., Houeto, J. L., Krystkowiak, P., Benabid, A. L., Cornu, P., Lagrange, C., Tezenas Du Montcel, S., Dormont, D., Grand, S., Blond, S. Detante, O., Pillon, B., Ardouin, C. Agid,Y., Destee,A., and Pollak, P. (2005) Bilateral deep-brain stimulation of the globus pallidus in primary generalized dystonia. N. Engl. J. Med. 352, 459-467.

Wichmann, T. (2000). A digital averaging method for removal of stimulus artifacts in neurophysiologic experiments. J. Neurosci. Methods 98, 57-62.

Windels, F., Bruet, N., Poupard, A., Feuerstein, C., Bertrand, A., and Savasta, M. (2003). Influence of the frequency parameter on extracellular glutamate and gamma-aminobutyric acid in substantia nigra and globus pallidus during electrical stimulation of subthalamic nucleus in rats. $J$. Neurosci. Res. 72, 259-267.

Windels, F., Bruet, N., Poupard, A., Urbain, N., Chouvet, G., Feuerstein, C., and Savasta, M. (2000). Effects of high frequency stimulation of subthalamic nucleus on extracellular glutamate and GABA in substantia nigra and globus pallidus in the normal rat. Eur. J. Neurosci. 12, 4141-4146.
Windels, F., Carcenac, C., Poupard, A., and Savasta, M. (2005). Pallidal origin of GABA release within the substantia nigra pars reticulata during high-frequency stimulation of the subthalamic nucleus. J. Neurosci. 25 , 5079-5086.

Wu, Y. R., Levy, R., Ashby, P., Tasker, R. R., and Dostrovsky, J. O. (2001). Does stimulation of the GPi control dyskinesia by activating inhibitory axons? Mov. Disord. 16, 208-216.

Xu, W., Russo, G. S., Hashimoto, T., Zhang, J., and Vitek, J. L. (2008). Subthalamic nucleus stimulation modulates thalamic neuronal activity. J. Neurosci 28, 11916-11924.

Yelnik, J., and Percheron, G. (1979). Subthalamic neurons in primates: a quantitative and comparative analysis. Neuroscience 4, 1717-1743.

Yianni, J., Bain, P., Giladi, N., Auca, M., Gregory, R., Joint, C., Nandi, D., Stein, J., Scott, R., and Aziz, T. (2003). Globus pallidus internus deep brain stimulation for dystonic conditions: a prospective audit. Mov. Disord. 18 , 436-442.

Conflict of Interest Statement: The authors declare that the research was conducted in the absence of any commercial or financial relationships that could be construed as a potential conflict of interest.

Received: 01 March 2011; paper pending published: 20 March 2011; accepted: 29 April 2011; published online: 13 May 2011. Citation: Devergnas A and Wichmann T (2011) Cortical potentials evoked by deep brain stimulation in the subthalamic area. Front. Syst. Neurosci. 5:30. doi: 10.3389/ fnsys.2011.00030

Copyright () 2011 Devergnas and Wichmann. This is an open-access article subject to a non-exclusive license between the authors and Frontiers Media SA, which permits use, distribution and reproduction in other forums, provided the original authors and source are credited and other Frontiers conditions are complied with. 